\title{
A Prospective Randomised Comparative Study of Monopolar Transurethral Resection of Prostate versus for Bipolar Resection of Prostate
}

\author{
${ }^{1}$ Nanjundarao Srinath, ${ }^{2}$ Sunil Jhulmaria, ${ }^{3}$ Gurvinder S Sethi
}

\begin{abstract}
We conducted a prospective randomized study to compare bipolar resection of prostate (BRP) with mono-polar transurethral resection of prostate (mTURP). A total of 120 patients of symptomatic benign prostatic hyperplasia $(\mathrm{BPH})$ were randomized into two groups and treated with bipolar resection of prostate with saline as irrigating fluid and monopolar TURP with $1.5 \%$ glycine as irrigation fluid. Pre- and post-op findings were recorded up to 6 months in all patients. Both the groups were comparable in age, prostate size regarding volume, international prostate symptom score (IPSS) and hemoglobin levels. The TURP group showed a statistically significant decline in $\mathrm{Hb}$ from the preoperative value. Postoperative serum sodium level was significantly low in the mTURP group than in the BRP group. We did not come across transurethral resection (TUR) syndrome in any patient. There was no significant difference in operation time, catheterization time, hospital stay among the two groups. Postoperative improvement in IPSS was similar. The peak urine flow (Q Max) was significantly high in bipolar resection group. Bipolar resection of prostate ha s lower intraoperative complications and improved results compared to monopolar TURP.
\end{abstract}

Keywords: Benign prostatic hyperplasia, Bipolar resection of prostate , TURP

How to cite this article:, Srinath N, Jhulmaria S, Sethi GS. A Prospective Randomised Comparative Study of Monopolar Transurethral Resection of Prostate versus for Bipolar Resection of Prostate. Int J Med Sci 2018;4(3):75-78.

Source of support: Nil

Conflict of interest: None

\section{INTRODUCTION}

Transurethral resection of the prostate (TURP) using monopolar cautery has been the gold standard for treatment of BPH with persistent symptoms of bladder

\footnotetext{
${ }^{1,3}$ Professor and HOD, ${ }^{2}$ Surgical Specialist

${ }^{1}$ Department of Urology, RajaRajeswari Medical College, Bengaluru, Karnataka, India.

${ }^{2}$ Command Hospital, Western Command, Chandimandir, Haryana, India

${ }^{3}$ Department of Urology, Command Hospital (AF), old Airport Road, Bangalore, Karnataka, India.

Corresponding Author: Nanjundarao Srinath, Professor and HOD, Department of Urology, RajaRajeswari Medical College, Bengaluru, Karnataka, India, e-mail: nsrinath1958@gmail.com
}

outlet obstruction. ${ }^{1}$ However, TURP is still associated with significant complications, such as TUR syndrome, hemorrhage, and strictures of the urethra. ${ }^{2}$ A bipolar resection of prostate which used the cutting effect by the creation of ionized plasma corona in an electrolyte solution (normal saline) offers a promising alternative to TURP with decreased physiological changes during surgery and likely decrease in complications. ${ }^{3}$

In this prospective randomized control study, the two procedures were compared in terms of efficacy and complications.

\section{MATERIALS AND METHODS}

We conducted a prospective randomized study on patients with a diagnosis of benign enlargement of prostate undergoing surgical treatment at a tertiary care hospital from May 2012 to June 2015. We recruited 120 patients with IPSS $>18$, residual volume more than $100 \mathrm{~mL}$, prostate volume 40 to $90 \mathrm{cc}$. Patients aged below 50 years, patients with urethral strictures, neurogenic bladder were excluded. Pre-op hemoglobin (Hb), IPSS score, ultrasound measured prostate volume, operative time, post-op $\mathrm{Hb}$, was recorded for all patients. Postoperatively the patients were re-evaluated at 1, 3 and 6 months with IPSS, postvoid residual urine and $Q$ Max.

\section{OPERATING TECHNIQUE}

Computer generated randomization into two groups divided the patients. In Group I ( $n=60)$ TURP was conducted with $26 \mathrm{~F}$ resectoscope using continuous flow with standard loop electrode using monopolar cautery(cutting $100 \mathrm{~W}$ and coagulation $80 \mathrm{~W}$ ) using $1.5 \%$ glycine as an irrigation fluid. In Group II ( $n=60)$, patients underwent PRP using plasma kinetic generator (TURIS, Olympus) with settings of $230 \mathrm{~W}$ cutting $75 \mathrm{~W}$ coagulation and $0.9 \%$ saline as irrigation fluid using $27^{\circ} \mathrm{F}$ continuous flow resectoscope (Olympus).

All operations were done under subarachnoid spinal anesthesia. A baseline blood sample $(2 \mathrm{~mL})$ used to be sent for estimation of serum sodium and potassium. These tests were repeated at 1-hour and the end of the procedure. Hemoglobin $(\mathrm{Hb})$ and hematocrit were studied before and after the procedure in all patients. Resection 
time, the volume of irrigation fluid used, the weight of the resected gland and duration of surgery were recorded.

The patient's vital parameters (pulse, blood pressure, oxygen saturation monitoring by pulse oximeter) were monitored, and clinical signs of TUR syndrome was watched for. Barnes method of resection was used in all our patients.

All patients received postoperative irrigation through 3 way Foley's catheter. The catheter was removed on postoperative Day 3 in all cases.

\section{RESULTS}

In the study, the majority of patients were in the age group 55 to 70 years. Age, prostate volume by ultrasound, maximum flow rate (Q Max, effective capacity of a bladder, after voiding residual urine, IPSS and pre-op hemoglobin $(\mathrm{Hb})$ were comparable. (Table 1$)$.

Operation time was longer in the BRP group. The weight of resected gland, volumes of irrigation and catheterization time were comparable between the two groups and not significant (Table 2).

The monopolar group had a greater fall of serum sodium (5.27 meq/L) compared to the BRP group (1.43 meq/L), and this was statistically highly significant between the groups ( $p=0.000$ ). However, TUR syndrome was not observed in both groups. The mTURP group showed a statistically highly significant decline in $\mathrm{Hb} 1.033 \mathrm{gm} \%$ (0.2617), p $=0.000$ from the preoperative value. In comparison, the bipolar group showed a smaller drop in hemoglobin 0.607 gm \% (0.1617). Hematocrit values showed a similar trend with the mTURP group recording a more significant fall from the preoperative value as compared to the BRP group (2.690, and 1.593, $\mathrm{p}<0.05$ respectively) (Table 3). However, none of the patients required a blood transfusion.

There were no significant complications other than clot retention for which cystoscopy and clot evacuation was needed in two patients in the monopolar group and one patient in the bipolar group.

Follow-up of patients (1,3 and 6 months postoperatively) demonstrated an improvement in I-PSS and Qmax in both the groups. The improvement in IPSS and drop in PVR was comparable in both groups (Tables 4 and 5). The Qmax at follow-up was significantly high in the bipolar group as compared to the monopolar group ( $\mathrm{p}<0.05$; Table 6).

Table 1: Preoperative parameters of patients participating in the study

\begin{tabular}{lll}
\hline & \multicolumn{2}{c}{ Mean $+S D$} \\
\cline { 2 - 3 } Parameters & $m T U R P(N=60)$ & $B R P(N=60)$ \\
\hline Age mean (range) in years & $66.5(60-74)$ & $68.1(61-80)$ \\
Prostate volume $(\mathrm{mL})$ & $64 \pm 8$ & $66 \pm 9$ \\
Q Max $(\mathrm{mL} / \mathrm{s})$ & $7.73(0.883)$ & $7.75(0.786)$ \\
Effective capacity $(\mathrm{mL})$ & $380(110)$ & $410(120)$ \\
Post void residual urine $(\mathrm{mL})$ & $240.18(65.854)$ & $220.65(43.414)$ \\
IPSS score & $28.53(3.963)$ & $29.40(4.507)$ \\
Hemoglobin & $13.803(0.8336)$ & $14.450(1.1500)$ \\
\hline
\end{tabular}

Table 2: Intraoperative variables between bipolar and monopolar groups

\begin{tabular}{llll}
\hline & $m$-TURP mean (SD) & BRP mean $(S D)$ & Significance \\
\hline Volume of irrigant (Ltrs) & $25.37(2.414)$ glycine & $25.53(2.193)$ saline & 0.781 \\
Resection time $(\mathrm{min})$ & $60.73(5.589)$ & $65.67(6.625)$ & 0.003 \\
Resected weight $(\mathrm{g})$ & $33.17(3.130)$ & $36.07(4.127)$ & 0.008 \\
Catheterisation time & $3.5(1.1)$ & $3.8(0.8)$ & 0.653 \\
\hline
\end{tabular}

Table 3: Comparison of mean change of electrolytes and hemoglobin between mTURP and PRP groups

\begin{tabular}{llll}
\hline & \multicolumn{2}{c}{ Mean change } & \\
\cline { 2 - 3 } Parameter & Glycine (Variance) & Saline (Variance) & 0.0001 \\
\hline Mean Sodium(meq/l) & $-5.27(1.081)$ & $-0.07(1.437)$ & 0.0002 \\
Mean Potassium(meq/l) & $+0.137(0.0765)$ & $+0.050(0.0900)$ & 0.000 \\
Mean Hb(gm\%) & $-1.033(0.2617)$ & $-0.607(0.1617)$ & 0.000 \\
Mean HCT & $2.690(0.5530)$ & $1.593(0.4777)$ & \\
\hline
\end{tabular}


A Prospective Randomised Comparative Study of Monopolar Transurethral Resection

Table 4: Comparison of IPSS between bipolar and monopolar groups

\begin{tabular}{llll}
\hline Time & Bipolar IPSS mean (SD) & Monopolar IPSS mean (SD) & $p$-value \\
\hline Pre-op & $28.53(3.963)$ & $29.40(4.507)$ & 0.432 \\
Post-op 1 month & $8.07(0.907)$ & $7.60(0.770)$ & 0.036 \\
Post-op 3 months & $8.63(0.890)$ & $7.60(0.770)$ & 0.048 \\
Post-op 6 months & $8.10(0.923)$ & $7.60(0.770)$ & 0.026 \\
\hline
\end{tabular}

Table 5: Comparison of PVR between bipolar and monopolar groups

\begin{tabular}{llll}
\hline Time & Bipolar PVR $(\mathrm{mL})$ & Mean $(S D)$ Monopolar PVR $(\mathrm{mL})$ & Mean $(S D) p$-value \\
\hline Pre-op & $240.18(65.854)$ & $220.65(43.414)$ & 0.268 \\
Post-op 1 month & $25.17(6.395)$ & $27.00(5.675)$ & 0.245 \\
Post-op 3 months & $26.53(5.296)$ & $27.80(5.442)$ & 0.365 \\
Post-op 6 months & $24.03(5.910)$ & $28.53(5.303)$ & 0.003 \\
\hline
\end{tabular}

Table 6: Comparison of $Q$ Max between bipolar and monopolar groups

\begin{tabular}{llll}
\hline Time & Bipolar Q Max $(\mathrm{mL} / \mathrm{s})$ & Mean $(\mathrm{SD})$ Monopolar Q Max $(\mathrm{mL} / \mathrm{s})$ & Mean $(\mathrm{SD}) \mathrm{p}$-value \\
\hline Pre-op & $7.73(.883)$ & $7.75(.786)$ & 0.931 \\
Post-op 1 month & $18.90(1.322)$ & $16.23(1.073)$ & 0.000 \\
Post-op 3 months & $18.93(1.172)$ & $16.37(0.999)$ & 0.000 \\
Post-op 6 months & $19.13(1.252)$ & $16.47(0.937)$ & 0.000 \\
\hline
\end{tabular}

\section{DISCUSSION}

The advent of fairly effective medical therapy for the lower urinary tract symptoms (LUTS) because of BPH in the form of alfa blockers and five alfa reductase inhibitors has led to decline in the surgical treatment of BPH. TURP continues to be the gold standard surgical procedure whenever there is an indication for surgery. ${ }^{1}$ Though TURP is a safe procedure (mortality $>0.25 \%$ ), it has significance. Intraoperative complications (blood loss, with a $2.5-8.6 \%$ transfusion rate, and TUR syndrome $)^{2} \mathrm{BRP}$ with use of normal physiological saline as irrigation fluid is promising in decreasing these complications.

In our randomized control study, we compared two groups of patients (sixty each) undergoing mTURP and BRP with glycine and saline as irrigating solutions respectively. The two groups had no statistically significant differences in baseline characteristics of patient's age and ASA status. Two senior consultant urologists performed the operations.

In this study, there was an increased time of resection in bipolar group (63.50 min,) more than monopolar group (54.67) which was statistically significant. This may have been due to the smaller loop of BRP resectoscope.

The mean duration of catheterization in both the groups was comparable. Hb fall after mTURP was higher than BRP indicating better hemostasis with BRP.

The current study showed that the BRP with normal saline as irrigating fluid showed negligible fall in serum
Na compared to mTURP with Glycine as irrigating fluid. $^{8}$

During our patient follow-up (1,3, and 6 months postoperatively) we found an improvement in IPSS and Qmax in both the groups. IPSS improvement was comparable in both groups. The Q-max at was significantly higher in the bipolar group as compared to the monopolar group.

The current study showed significantly higher improvement in peak urinary flow postoperatively in patients undergoing BRP than in mTURP group.

\section{CONCLUSION}

The present study shows that the bipolar system is a promising technique in the surgical management of $\mathrm{BPH}$. Our results demonstrate that bipolar transurethral resection of prostate causes less drop in sodium, hemoglobin, and level than monopolar transurethral resection of prostate. Similar findings wereseen some earlier studies. ${ }^{4-6}$ However, it takes a longer resection time. Bipolar saline TURP is a safer option in patients with large prostates with multiple comorbidities.

Our study suggests that at 6 months of follow-up bipolar TURP is equivalent to monopolar in terms of efficacy. The procedures also have a comparable complication profile. However, the use of normal saline for irrigation improves the safety of procedure. Our study indicates that BRP is clinically as effective as mTURP with a better safety profile. 


\section{REFERENCES}

1. Mamoulakis C, Trompetter M, de la Rosette J Bipolar transurethral resection of the prostate: the "golden standard" reclaims its leading position. Curr Opin Urol 2009;19: 26-32.

2. Reich O, Gratzke C, Bachmann A Morbidity, mortality and early outcome of transurethral resection of the prostate: a prospective multicenter evaluation of 10,654 patients. J Urol 2008;180: 246-249.

3. Issa MM: Technological advances in transurethral resection of the prostate: bipolar versus monopolar TURP J Endourol 2008;22:1587-1595.
4. Singh H, Desai MR, Shrivastav P, Vani K. Bipolar versus monopolar transurethral resection of prostate: randomized controlled study J Endourol 2005;19:333-338.

5. Smith D, Khoubehi B, Patel A. Bipolar electrosurgery for benign prostatic hyperplasia: transurethral electrovapor $\rightarrow$ ization and resection of the prostate. Curr. Opin. Urol. 2005;15: 95-100.

6. Treparier CA, Lessard MR, Brochu J, Turcotte G. Another feature of TURP syndrome: hyperglycaemia and lactic acidosis caused by massive absorption of sorbitol. Br J. Anaesth 2001;87:316-319. 